\title{
The relationship between the tumour stroma percentage, clinicopathological characteristics and outcome in patients with operable ductal breast cancer
}

F J A Gujam*,1, J Edwards², Z M A Mohammed ${ }^{1}$, J J Going ${ }^{3}$ and D C McMillan ${ }^{1}$

${ }^{1}$ Academic Unit of Surgery, College of Medical, Veterinary and Life Sciences-University of Glasgow, Royal Infirmary, Glasgow G31 2ER, UK; ${ }^{2}$ Unit of Experimental Therapeutics, Institute of Cancer, College of Medical, Veterinary and Life Sciences-University of Glasgow, Western Infirmary, Glasgow, UK and ${ }^{3}$ University Section of Pathology, College of Medical, Veterinary and Life Sciences-University of Glasgow, Southern General Hospital, Glasgow G51 4TF, UK

Background: The percentage of tumour stroma (TSP) has recently been reported to be a novel independent predictor of outcome in patients with a variety of common solid organ tumours. The aim of this study was to examine the relationship between TSP, clinicopathological characteristics and outcome in patients with invasive ductal breast cancer, in particular node negative and triple negative disease.

Methods: A total of 361 patients with primary operable invasive ductal breast cancer were included in this study. The TSP was assessed visually on the haematoxylin and eosin-stained tissue sections. With a cutoff value of $50 \%$ TSP, patients with $\leqslant 50 \%$ stroma were classified as the low-TSP group and those with $>50 \%$ stroma were classified as the high-TSP group.

Results: A total of 109 (30\%) patients had high TSP. Patients with high TSP were old age $(P=0.035)$, had more Her-2-positive tumours $(P=0.029)$, low-grade tumour inflammatory infiltrate $(P=0.034)$, low $C D 68+$ macrophage infiltrate $(P<0.001)$, low $C D 4+$ $(P=0.023)$ and low $C D 8+$ T-lymphocytes infiltrate $(P=0.017)$, tumour recurrence $(P=0.015)$ and shorter cancer-specific survival $(P<0.001)$. In node-negative patients $(n=207)$, high TSP was associated with low CD68 + macrophage infiltrate $(P=0.001)$, low CD4 $+(P=0.040)$ and low CD8 + T-lymphocytes infiltrate $(P=0.016)$ and shorter cancer-specific survival $(P=0.005)$. In triple negative patients $(n=151)$, high TSP was associated with high tumour grade $(P=<0.001)$, lymph node positivity $(P=0.027)$, low CD68 + macrophage infiltrate $(P=0.011)$ and shorter cancer-specific survival $(P=0.035)$. The 15-year cancer-specific survival rate was $79 \%$ vs $21 \%$ in the low-TSP group vs high-TSP group. In multivariate survival analysis, a high TSP was associated with reduced cancer-specific survival in the whole cohort $(P=0.001)$, node-negative patients $(P=0.007)$ and those who received systemic adjuvant therapy $(P=0.021)$, independent of other pathological characteristics including host inflammatory response. However, TSP was not an independent prognostic factor for triple negative patients $(P=0.151)$.

Conclusions: A high TSP in primary operable invasive ductal breast cancer was associated with recurrence and poorer long-term survival. The inverse relation with the tumour inflammatory infiltrate highlights the importance of the amount of tumour stroma on immunological response in patients with primary operable ductal breast cancer. Implementing this simple and reproducible parameter in routine pathological examination may help optimise risk stratification in patients with invasive ductal breast cancer.

*Correspondence: Dr FJA Gujam; E-mail: f.gujam.1@research.gla.ac.uk

Received 1 February 2014; revised 7 April 2014; accepted 30 April 2014; published online 29 May 2014

(c) 2014 Cancer Research UK. All rights reserved 0007 - 0920/14 
Breast cancer is the commonest cancer and the leading cause of cancer death in women. It accounts for approximately 10th of all new cancers and $25 \%$ of all female cancers (Ferlay et al, 2010). With the advent of a screening programme, more than $70 \%$ of newly diagnosed invasive tumours present without nodal involvement (Health and Consumer Protection Directorate-General, 2006) and those women have $88 \% 10$-year disease-free survival rate (Mirza et al, 2002).

In recent years, the simple concept that tumour progression depends solely on the intrinsic properties of cancer cells has recently given way to a more complex paradigm in which tumour progression depends on the interaction between tumour and host cells (Colotta et al, 2009; Hanahan and Weinberg, 2011). Recent evidence suggests that the tumour stroma profoundly influences tumour growth, angiogenesis and dissemination. Tumour stroma is thought to promote tumourigenesis by different mechanisms including remodelling of the extracellular matrix, suppression of immune response and alterations in stromal regulatory pathways affecting the motility and aggressiveness of cancer cells (Kim et al, 2005; Hu and Polyak, 2008; Cirri and Chiarugi, 2012).

Recently, it has been reported that tumour stroma has prognostic value in patients with colorectal (Mesker et al, 2007; West et al, 2010; Huijbers et al, 2013, Park et al, 2014) and oesophageal cancers (Staal et al, 2010; Wang et al, 2012). Also, the percentage of tumour stroma has been recently reported to have prognostic value in patients with triple negative (de Kruijf et al, 2011; Moorman et al, 2012) and node-negative breast cancer (Dekker et al, 2013). However, the relationship with other important determinants of outcome such as the lymphovascular invasion, necrosis and tumour inflammatory cell infiltrate is unclear.

Therefore, the aim of this study was to examine the relationship between the percentage of tumour to stroma, host inflammatory response, clinicopathological characteristics and outcome in patients with early breast cancer, in particular node negative and triple negative disease.

\section{MATERIALS AND METHODS}

Patients. Patients presenting with primary operable invasive ductal breast cancer at Royal Infirmary, Western Infirmary and Stobhill Hospital, Glasgow, between 1995 and 1998 were studied $(n=361)$. Clinicopathological data including age, histological tumour type, grade, tumour size, lymph node status, type of surgery and use of adjuvant treatment (chemotherapy, hormonal therapy and/or radiotherapy) were retrieved from the routine reports. ER, PR and Her-2 status were performed as previously described and the results of visual scoring were used in this study
(Mohammed et al, 2012a,b). Tumour necrosis, general inflammatory infiltrate, CD68 + macrophage infiltrate, CD4+ T-lymphocyte infiltrate, CD8 + T-lymphocyte infiltrate were performed as previously described (Ikpatt et al, 2002; Klintrup et al, 2005; Mohammed et al, 2012c,2013). Ki67 was performed as previously described (Mohammed et al, 2012d).

In the present study, the proportion of patients with ERnegative tumours was relatively high in the TMA. This enabled specific examination of the prognostic value of tumour characteristics in a sub-cohort of patients with ER-negative ductal breast. Also, the patients included in this study did not receive neoadjuvant therapy or adjuvant anti-HER-2 therapy. The inclusion of ductal breast cancers only was to limit the potential confounding effects of other tumour types on the analysis in the present study.

Patients were routinely followed up following surgery. Date and cause of death was cross-checked with the cancer registration system and the Registrar General (Scotland). Death records were complete until 31st of May 2013 and that served as the censor date. Cancer recurrence was measured from the date of primary surgery until the date of first recurrence of breast cancer. Cancer-specific survival was measured from the date of primary surgery until the date of death from breast cancer.

Institutional Review Board approval for the use of human tissue in this study was given by the Research Ethics Committee of the North Glasgow University Hospitals NHS Trust.

Slide scanning and assessment of tumour stroma. The haematoxylin and eosin tumour sections stained according to standard histological protocols were scanned using a Hamamatsu NanoZoomer (Welwyn Garden City, Hertfordshire, UK) at objective magnification $\times 20$. Visualisation and image analysis assessment was carried out using Slidepath Digital Image Hub, version 4.0.1, (Slidepath, Leica Biosystems, Milton Keynes, UK). Visual assessment of TSP was performed on a high-definition monitor and was carried out at the most invasive tumour area according to previously described criteria (Mesker et al, 2007). As Slidepath provides different levels of magnification similar to a conventional microscope, the most invasive tumour area to be analysed was identified visually and selected using a $\times 4$ or $\times 5$ magnification. The magnification was then set to $\times 10$ at the selected area where both stroma and tumour tissue were available. Tumour cells must be present at all borders of the image field (north-east-southwest) (Figure 1). When necrotic and mucinous tissue was present within the selected area, the mucinous tissue was visually excluded for the scoring. Scoring percentages were given per 10-fold $(10,20$, $30 \%$ etc.).

Cutoff at 50\% TSP was used as described in previous reports (Mesker et al, 2007; de Kruijf et al, 2011) i.e., low stroma tumours
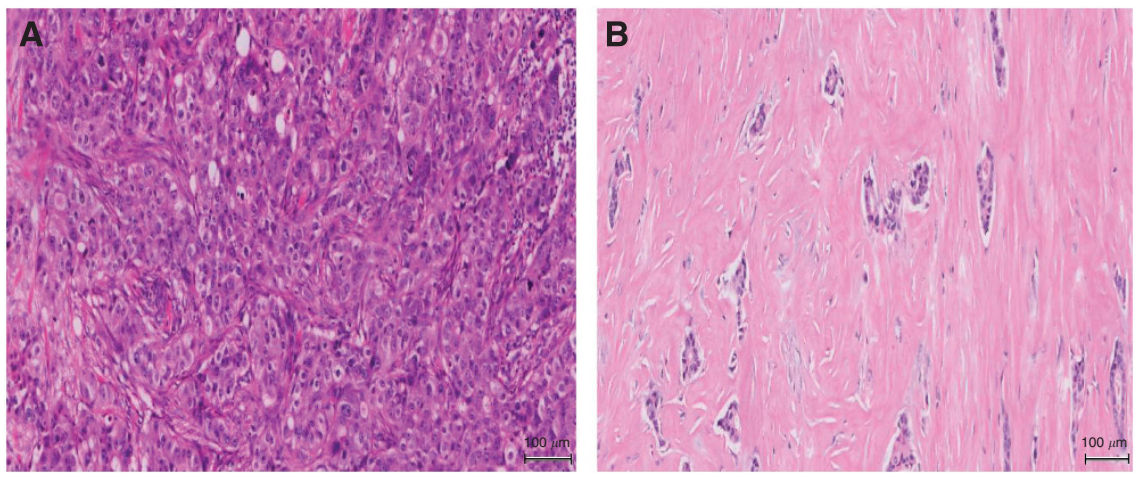

Figure 1. Haematoxylin and eosin-stained sections of invasive ductal breast tumours. (A) Tumour with low stroma (10\%); (B) tumour with high stroma (80\%). Magnificatiom $\times 10$ objective and $100-\mu \mathrm{m}$ scale. 
were the presence of tumour stroma in $\leqslant 50 \%$ of tumour area (Figure 1A), whereas high stroma tumours were the presence of tumour stroma in $>50 \%$ of tumour area (Figure $1 \mathrm{~B}$ ).

A total of 40 specimens were independently estimated for TSP by two observers (FJAG and JE) blinded to the patient outcome and the other observer's score. The ICCC was 0.83 indicating excellent agreement. The author (FJAG) then scored the rest of slides.

Statistical analysis. Consistency between the observers was analysed using the ICCC value. Inter-relationships between variables were assessed using contingency table analysis with the $\chi^{2}$ test for trend as appropriate. Univariate and multivariate survival analysis were performed using the Kaplan-Meier analysis and Cox proportional hazards model. A stepwise backward procedure was used to derive a final model of the variables that had a significant independent relationship with survival. All statistical analyses were two-sided and significance defined as $P$-value $<0.05$. Deaths up to May 2013 were included in the analysis. All statistical analysis was performed using the SPSS software version 19 (SPSS Inc., Chicago, IL, USA).

\section{RESULTS}

Clinicopathological characteristics. Table 1 shows clinicopathological characteristics of patients $(n=361)$. The majority were older than 50 years (65\%), had a grade III carcinoma (54\%) equal or smaller than $2 \mathrm{~cm} \mathrm{(51 \% )} \mathrm{with} \mathrm{no} \mathrm{axillary} \mathrm{lymph} \mathrm{node}$ involvement (57\%). The majority had ER-negative tumours (54\%), PR-negative tumours (66\%) and Her2-negative tumours $(80 \%)$. A total of $30 \%$ had lymphovascular invasion and the majority had a high-grade tumour necrosis (64\%). In total, $60 \%$ had low-grade general tumour inflammatory infiltrate with the cellular inflammatory infiltrates (CD68 + macrophage infiltrate, CD4 + T-lymphocyte infiltrate and CD8 + T-lymphocyte infiltrate) presented as tertiles (Table 1). In all, 81 (22\%) patients received only hormonal therapy, 144 (40\%) received only chemotherapy and 45 (13\%) received both, and data on seven cases were not available.

The minimum follow-up of survivors was 142 months and the median follow-up was 168 months. During follow-up, 89 patients developed recurrence (25\%), 172 patients died, $27 \%$ died of their cancer.

TSP. For all patients, TSP was evaluated on one section derived from the most invasive part of the tumour. The tumour specimens showed variety in TSP ranging from very solid tumours with little stromal involvement (Figure 1A) to tumours with large areas of stromal proliferation scattered with single and grouped tumour cells (Figure 1B). In total, $252(70 \%)$ patients had low TSP $(\leqslant 50 \%$ stroma) and $109(30 \%)$ patients had high TSP (>50\% stroma). In node-negative patients, 153 (74\%) patients had low TSP and 54 (26\%) had high TSP. In triple negative patients, 115 (76\%) patients had low TSP and 36 (24\%) had high TSP.

Association of TSP with clinicopathological variables and outcome. The relationship between TSP, clinicopathological variables and host inflammatory response is shown in Tables 2-4. Patients with high TSP were old age women $(P=0.035)$, had more Her-2-positive tumours $(P=0.029)$, low-grade general inflammatory infiltrate $(P=0.034)$, low CD68 + macrophage infiltrate $(P<0.001)$, low $\mathrm{CD} 4+(P=0.023)$ and low $\mathrm{CD} 8+$ T-lymphocytes infiltrate $(P=0.017)$, had tumour recurrence $(P=0.015)$ and shorter cancer-specific survival $(P=0.001)$. In node-negative patients $(n=207)$, a high TSP was associated with low CD68 + macrophage infiltrate $(P=0.001)$, low $\mathrm{CD} 4+$ $(P=0.040)$ and low CD8 + T-lymphocytes infiltrate $(P=0.016)$ and shorter cancer-specific survival $(P=0.005)$. In triple negative patients $(n=151)$, a high TSP was strongly associated with high tumour grade $(P=<0.001)$, lymph node positivity $(P=0.027)$, low CD68 + macrophage infiltrate $(P=0.011)$, low $\mathrm{CD} 4+$ $(P=0.049)$ and tended to have low CD8 + T-lymphocytes infiltrate $(P=0.071)$ and shorter cancer-specific survival

\begin{tabular}{|c|c|}
\hline Clinicopathological characteristics & Patients (n) \\
\hline Age $(\leqslant 50 />50$ years $)$ & $125(35 \%) / 236(65 \%)$ \\
\hline Size $(\leqslant 20 / 21-50 />50 \mathrm{~mm})$ & $185(51 \%) / 163(45 \%) / 13(4 \%)$ \\
\hline Grade (I/II/III) & $48(13 \%) / 124(34 \%) / 189(52 \%)$ \\
\hline Involved lymph node $(0 / 1-3 />3)$ & $207(57 \%) / 120(33 \%) / 34(9 \%)$ \\
\hline Oestrogen receptor status (no/yes) & $194(54 \%) / 167(46 \%)$ \\
\hline Progesterone status (no/yes) & $238(66 \%) / 123(34 \%)$ \\
\hline Her-2 status (no/yes) & $290(80 \%) / 71(20 \%)$ \\
\hline Lymphovascular invasion (no/yes) & $251(70 \%) / 110(30 \%)$ \\
\hline Tumour necrosis (no/yes) & $131(36 \%) / 230(64 \%)$ \\
\hline General inflammatory infiltrate (low/high) & $215(60 \%) / 146(40 \%)$ \\
\hline CD68 + macrophage infiltrate (tertiles) & $82(23 \%) / 115(32 \%) / 103(29 \%) / 61(17 \%)^{a}$ \\
\hline CD4 + T-lymphocyte infiltrate (tertiles) & $132(37 \%) / 112(31 \%) / 117(32 \%)$ \\
\hline CD8 + T-lymphocyte infiltrate (tertiles) & $121(34 \%) / 118(33 \%) / 122(34 \%)$ \\
\hline Tumour stroma percentage $(\leqslant 50 \% />50 \%)$ & $252(70 \%) / 109$ (30\%) \\
\hline Loco-regional therapy (Lumpectomy + radiotherapy/mastectomy + radiotherapy) & $130(36 \%) / 231(64 \%)$ \\
\hline Systemic adjuvant therapy (hormonal/hormonal + chemotherapy/chempotherapy/none) & $81(22 \%) / 45(13 \%) / 144(40 \%) / 84(23 \%) / 7(2 \%)^{a}$ \\
\hline Tumour recurrence (no/yes) & $272(75 \%) / 89(25 \%)$ \\
\hline Alive/cancer death/non-cancer death & $189(52 \%) / 97(27 \%) / 75$ (21\%) \\
\hline
\end{tabular}


Table 2. The inter-relationship between clinicopathological characteristics and tumour stroma percentage in patients with invasive ductal breast cancer $(n=361)$

\begin{tabular}{|c|c|c|c|}
\hline & $\begin{array}{c}\text { Tumour stroma } \\
\text { percentage } \leqslant 50 \quad n=252(70 \%)\end{array}$ & $\begin{array}{c}\text { Tumour stroma } \\
\text { percentage }>50 n=109(30 \%)\end{array}$ & $P$-value \\
\hline Age $(\leqslant 50 />50$ years $)$ & $96 / 156$ & $29 / 80$ & 0.035 \\
\hline Size $(\leqslant 20 / 21-50 />50 \mathrm{~mm})$ & $136 / 108 / 8$ & $49 / 55 / 5$ & 0.109 \\
\hline Grade (I/II/III) & $35 / 77 / 140$ & $13 / 47 / 49$ & 0.289 \\
\hline Involved lymph node $(0 / 1-3 />3)$ & $153 / 78 / 21$ & $54 / 42 / 13$ & 0.052 \\
\hline Oestrogen receptor status (no/yes) & $138 / 114$ & $56 / 53$ & 0.554 \\
\hline Progesterone status (no/yes) & $168 / 84$ & $70 / 39$ & 0.653 \\
\hline Her-2 status (no/yes) & $210 / 42$ & $80 / 29$ & 0.029 \\
\hline Lymphovascular invasion (no/yes) & $180 / 72$ & $71 / 38$ & 0.234 \\
\hline Tumour necrosis (no/yes) & $91 / 161$ & $40 / 69$ & 0.915 \\
\hline General inflammatory infiltrate (low/high) & $141 / 111$ & $74 / 35$ & 0.034 \\
\hline CD68 + macrophage infiltrate (tertiles) & $40 / 84 / 80$ & $42 / 31 / 23$ & $<0.001$ \\
\hline CD4 + T-lymphocyte infiltrate (tertiles) & $66 / 111 / 75$ & $36 / 54 / 19$ & 0.023 \\
\hline CD8 + T-lymphocyte infiltrate (tertiles) & $71 / 80 / 101$ & $73 / 46 / 26$ & 0.017 \\
\hline $\begin{array}{l}\text { Loco-regional therapy (Lumpectomy + radiotherapy/ } \\
\text { mastectomy + radiotherapy) }\end{array}$ & $93 / 159$ & $37 / 72$ & 0.591 \\
\hline $\begin{array}{l}\text { Systemic adjuvant therapy (hormonal/hormonal + } \\
\text { chemotherapy/chempotherapy/none) }\end{array}$ & $55 / 35 / 97 / 61$ & $30 / 21 / 35 / 23$ & 0.104 \\
\hline Tumour recurrence (no/yes) & $199 / 53$ & $73 / 36$ & 0.015 \\
\hline Alive/cancer death/non-cancer death & $151 / 55 / 46$ & $38 / 42 / 29$ & $<0.001$ \\
\hline Cancer-specific survival (months) ${ }^{a}$ & $176(168-186)$ & $144(128-160)$ & $<0.001$ \\
\hline
\end{tabular}

Table 3. The inter-relationship between clinicopathological characteristics and tumour stroma percentage in node-negative patients $(n=207)$

\begin{tabular}{|c|c|c|c|}
\hline & $\begin{array}{l}\text { Tumour stroma percentage } \\
\quad(\leqslant 50) n=153(74 \%)\end{array}$ & $\begin{array}{l}\text { Tumour stroma percentage } \\
\qquad(>50) n=54(26 \%)\end{array}$ & $P$-value \\
\hline Age $(\leqslant 50 />50$ years $)$ & $54 / 99$ & $13 / 41$ & 0.131 \\
\hline Size $(\leqslant 20 / 21-50 />50 \mathrm{~mm})$ & $90 / 60 / 3$ & $30 / 23 / 1$ & 0.709 \\
\hline Grade (I/II/III) & $26 / 45 / 82$ & $8 / 28 / 18$ & 0.123 \\
\hline Oestrogen receptor status (no/yes) & $84 / 69$ & $26 / 28$ & 0.394 \\
\hline Progesterone status (no/yes) & $103 / 50$ & $35 / 19$ & 0.738 \\
\hline Her-2 status (no/yes) & $128 / 25$ & $41 / 13$ & 0.208 \\
\hline Lymphovascular invasion (no/yes) & $123 / 25$ & $41 / 13$ & 0.488 \\
\hline Tumour necrosis (no/yes) & $62 / 91$ & $21 / 33$ & 0.488 \\
\hline General inflammatory infiltrate (low/high) & $89 / 64$ & $39 / 15$ & 0.068 \\
\hline CD68 + macrophage infiltrate (tertiles) & $27 / 49 / 46$ & $24 / 13 / 10$ & 0.001 \\
\hline CD4 + T-lymphocyte infiltrate (tertiles) & $36 / 67 / 50$ & $17 / 28 / 9$ & 0.040 \\
\hline CD8 + T-lymphocyte infiltrate (tertiles) & $41 / 52 / 60$ & $22 / 20 / 12$ & 0.016 \\
\hline Recurrence status (no/yes) & $130 / 23$ & $41 / 13$ & 0.133 \\
\hline $\begin{array}{l}\text { Loco-regional therapy (Lumpectomy + radiotherapy/ } \\
\text { mastectomy + radiotherapy) }\end{array}$ & $65 / 88$ & $24 / 30$ & 0.803 \\
\hline $\begin{array}{l}\text { Systemic adjuvant therapy (hormonal/hormonal + } \\
\text { chemotherapy/ chemotherapy/none) }\end{array}$ & $35 / 12 / 55 / 49$ & $15 / 8 / 16 / 15$ & 0.251 \\
\hline Alive/cancer death/non-cancer death & $102 / 20 / 31$ & $124 / 35 / 48$ & 0.002 \\
\hline Cancer-specific survival (months) ${ }^{a}$ & $192(183-201)$ & $164(144-184)$ & 0.005 \\
\hline
\end{tabular}


$(P=0.035)$. A high TSP was not associated with hormonal status, tumour necrosis and lymphovascular invasion.

Sub-group analysis of the relationship between the TSP and Ki67 in different patient groups was performed (Table 5). Only $59 \%$ of patients from the whole cohort, $44 \%$ from node-negative group and 65\% from triple negative group had Ki67 information available. There was no significant statistical difference between high-TSP and low-TSP groups in all sub-cohorts.

The 15 -year cancer-specific survival rate was $79 \%$ vs $21 \%$ in the low-TSP group $v s$ high-TSP group. Kaplan-Meier survival curves show that high TSP was significantly associated with poorer cancer-specific survival in the whole cohort $(P<0.001)$, in nodenegative patients $(P=0.005)$ and in triple negative patients $(P=0.035)$ (Figures $2 \mathrm{~A}-\mathrm{C})$. In multivariate survival analysis, a high TSP was associated with reduced cancer-specific survival independent of other variables in the whole cohort (HR 2.12, 95\% CI 1.37-3.29, $P=0.001$ ) and in node-negative patients (HR 3.11, 95\% CI 1.53-6.33, $P=0.007)$ but not in triple negative patients $(P=0.151)$ (Tables 6-8).

The relationship between TSP, clinicopathological characteristics and survival in patients who underwent adjuvant systemic treatment was examined. In total, 270 (75\%) patients received adjuvant systemic treatment. A high TSP was associated with shorter cancer-specific survival following adjuvant treatment in univariate analysis (HR 2.04, 95\% CI 1.29-3.22, $P=0.002$ ). In multivariate analysis, a high TSP was associated with reduced cancer-specific survival (HR 1.86, 95\% CI 1.10-3.15, $P=0.021$ ), independent of PR status, lymph node involvement, tumour necrosis and CD68 + T-lymphocyte infiltrate.

\section{DISCUSSION}

The results of the present study show that high TSP was consistently associated with low tumour inflammatory infiltrate. Furthermore, TSP was associated with poorer outcome in the whole cohort and in patients with node-negative disease with longterm follow-up. Taken together, the present results highlight the importance of the stroma in the tumour microenvironment and its impact on outcome.

Consistent with previous reports, the role of tumour stroma in breast cancer (de Kruijf et al, 2011; Moorman et al, 2012; Dekker et al, 2013) survival in the present study was significantly shorter in patients with high-TSP tumours. However, TSP was not independently associated with survival in triple negative patients, whereas de Kruijf et al (2011) reported that TSP was indeed a significant prognostic factor. The difference between these findings might be attributed to the differences in patients' characteristics or might be

Table 4. The inter-relationship between clinicopathological characteristics and tumour stroma percentage in triple negative patients with invasive ductal breast cancer $(n=151)$

\begin{tabular}{|c|c|c|c|}
\hline & $\begin{array}{c}\text { Tumour stroma } \\
\text { percentage } \leqslant 50 \quad n=115(76 \%)\end{array}$ & $\begin{array}{c}\text { Tumour stroma } \\
\text { percentage }>50 n=36(24 \%)\end{array}$ & $P$-value \\
\hline Age $(\leqslant 50 />50$ years $)$ & $49 / 66$ & $14 / 22$ & 0.694 \\
\hline Size $(\leqslant 20 / 21-50 />50 \mathrm{~mm})$ & $62 / 49 / 4$ & $15 / 18 / 7$ & 0.127 \\
\hline Grade (I/IIIIIII) & $0 / 17 / 98$ & $3 / 11 / 22$ & $<0.001$ \\
\hline Involved lymph node $(0 / 1-3 />3)$ & $68 / 30 / 10$ & $15 / 20 / 8$ & 0.027 \\
\hline Lymphovascular invasion (no/yes) & $77 / 38$ & $20 / 16$ & 0.214 \\
\hline Tumour necrosis (no/yes) & $28 / 87$ & $9 / 27$ & 0.931 \\
\hline General inflammatory infiltrate (low/high) & $44 / 71$ & $19 / 17$ & 0.124 \\
\hline CD68 + macrophage infiltrate (tertiles) & $19 / 28 / 36$ & $14 / 5 / 7$ & 0.011 \\
\hline CD4 + T-lymphocyte infiltrate (tertiles) & $19 / 48 / 48$ & $11 / 15 / 10$ & 0.049 \\
\hline CD8 + T-lymphocyte infiltrate (tertiles) & $25 / 34 / 56$ & $11 / 14 / 11$ & 0.071 \\
\hline Tumour recurrence (no/yes) & $83 / 32$ & $21 / 15$ & 0.119 \\
\hline $\begin{array}{l}\text { Loco-regional therapy (Lumpectomy + radiotherapy/ } \\
\text { mastectomy + radiotherapy) }\end{array}$ & $41 / 74$ & $18 / 18$ & 0.125 \\
\hline $\begin{array}{l}\text { Systemic adjuvant therapy (hormonal/hormonal + } \\
\text { chemotherapy/chempotherapy/none) }\end{array}$ & $13 / 7 / 67 / 25$ & $6 / 4 / 19 / 7$ & 0.296 \\
\hline Alive/cancer death/non-cancer death & $64 / 33 / 18$ & $13 / 16 / 7$ & 0.103 \\
\hline Cancer-specific survival (months) $^{a}$ & $176(167-185)$ & $147(133-163)$ & 0.035 \\
\hline
\end{tabular}

Table 5. The inter-relationship between tumour stroma percentage and Ki67 in patients with invasive ductal breast cancer

\begin{tabular}{|c|c|c|c|}
\hline All patients $(n=214)$ & $\begin{array}{l}\text { Tumour stroma percentage } \leqslant 50 \\
\qquad n=115(76 \%)\end{array}$ & $\begin{array}{l}\text { Tumour stroma percentage }>50 \\
\qquad n=36(24 \%)\end{array}$ & $P$-value \\
\hline Ki67 (low/high) & $122 / 27$ & $54 / 11$ & 0.833 \\
\hline Node-negative patients $(n=120)$ & 89 (74\%) & $31(26 \%)$ & \\
\hline Ki67 (low/high) & $75 / 14$ & $28 / 3$ & 0.407 \\
\hline Triple negative patients $(n=99)$ & $n=76(77 \%)$ & $n=23(23 \%)$ & \\
\hline Ki67 (low/high) & $61 / 15$ & $19 / 4$ & 0.803 \\
\hline
\end{tabular}



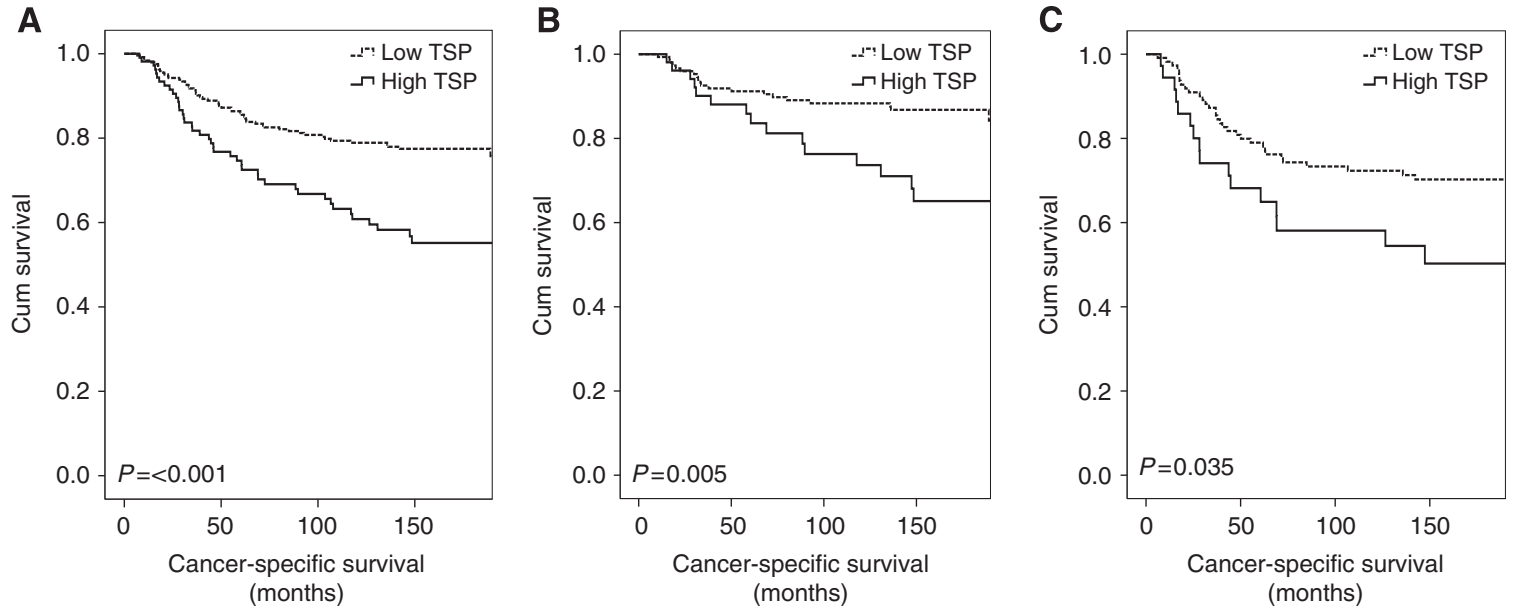

Figure 2. Kaplan-Meier survival curves Kaplan-Meier survival curves (log rank) of cancer-specific survival for tumour stroma percentage in the whole cohort (A), Node-negative patients (B) and triple negative patients (C).

Table 6. The relationship between clinicopathological characteristics and cancer-specific survival in patients with invasive ductal breast cancer $(n=361)$

\begin{tabular}{|c|c|c|c|c|}
\hline & \multicolumn{2}{|c|}{ Univariate analysis } & \multicolumn{2}{|c|}{ Multivariate analysis } \\
\hline & $\begin{array}{l}\text { Hazard ratio ( } 95 \% \\
\text { confidence interval) }\end{array}$ & $P$-value & $\begin{array}{l}\text { Hazard ratio ( } 95 \% \\
\text { confidence interval) }\end{array}$ & $P$-value \\
\hline Age $(\leqslant 50 />50$ years $)$ & $0.97(0.64-1.46)$ & 0.881 & & \\
\hline Size $(\leqslant 20 / 21-50 />50 \mathrm{~mm})$ & $2.17(1.54-3.07)$ & $<0.001$ & & 0.142 \\
\hline Grade (I/II/III) & $1.85(1.3-2.58)$ & $<0.001$ & $1.72(1.18-2.51)$ & 0.005 \\
\hline Involved lymph node $(0 / 1-3 />3)$ & $1.97(1.51-2.56)$ & $<0.001$ & $1.97(1.46-2.66)$ & $<0.001$ \\
\hline Oestrogen receptor status (no/yes) & $0.52(0.34-0.79)$ & 0.002 & & 0.240 \\
\hline Progesterone status (no/yes) & $0.44(0.32-0.82)$ & 0.006 & & 0.184 \\
\hline Her2 status (no/yes) & $1.44(0.88-2.35)$ & 0.145 & & \\
\hline Lymphovascular invasion (no/yes) & $2.07(1.39-3.09)$ & $<0.001$ & & 0.864 \\
\hline Tumour necrosis (no/yes) & $1.97(1.29-2.99)$ & 0.002 & $2.49(1.42-4.39)$ & 0.001 \\
\hline General inflammatory infiltrate (low/high) & $1.15(0.77-1.73)$ & 0.482 & & \\
\hline CD68 + T-lymphocyte infiltrate (tertiles) & $0.73(0.55-0.96)$ & 0.025 & & 0.174 \\
\hline CD4 + T-lymphocyte infiltrate (tertiles) & $0.46(0.23-1.70)$ & 0.075 & & \\
\hline CD8 + T-lymphocyte infiltrate (tertiles) & $0.64(0.49-0.82)$ & $<0.001$ & $0.66(0.46-0.80)$ & 0.002 \\
\hline $\begin{array}{l}\text { Loco-regional therapy (Lumpectomy + radiotherapy/ } \\
\text { mastectomy + radiotherapy) }\end{array}$ & $2.01(1.27-3.19)$ & 0.003 & & 0.535 \\
\hline $\begin{array}{l}\text { Systemic adjuvant therapy (hormonal/hormonal + } \\
\text { chemotherapy/chempotherapy/none) }\end{array}$ & $1.15(0.71-1.87)$ & 0.573 & & \\
\hline Tumour stroma percentage ( $\leqslant 50 \% />50 \%)$ & $1.89(1.26-2.82)$ & $<0.001$ & $2.12(1.37-3.29)$ & 0.001 \\
\hline \multicolumn{5}{|l|}{ Systemic adjuvant therapy $(n=207)$} \\
\hline Size $(\leqslant 20 / 21-50 />50 \mathrm{~mm})$ & $1.44(0.96-2.15)$ & 0.080 & & 0.462 \\
\hline Grade (I/II/III) & $1.66(1.13-2.43)$ & 0.010 & & 0.256 \\
\hline Involved lymph node $(0 / 1-3 />3)$ & $1.78(1.31-2.40)$ & $<0.001$ & $1.99(1.41-2.82)$ & $<0.001$ \\
\hline Oestrogen receptor status (no/yes) & $0.47(0.29-0.76)$ & 0.002 & & 0.181 \\
\hline Progesterone status (no/yes) & $0.47(0.27-0.83)$ & 0.009 & $0.49(0.26-0.89)$ & 0.020 \\
\hline Lymphovascular invasion (no/yes) & $1.99(1.23-3.15)$ & 0.003 & & 0.160 \\
\hline Tumour necrosis (no/yes) & $2.53(1.41-4.52)$ & 0.002 & $2.63(1.40-4.96)$ & 0.003 \\
\hline CD68 + T-lymphocyte infiltrate (tertiles) & $0.63(0.46-0.86)$ & 0.004 & $0.61(0.43-8.44)$ & 0.003 \\
\hline CD8 + T-lymphocyte infiltrate (tertiles) & $0.78(0.56-1.03)$ & 0.841 & & \\
\hline $\begin{array}{l}\text { Loco-regional therapy (Lumpectomy + radiotherapy/ } \\
\text { mastectomy + radiotherapy) }\end{array}$ & $1.93(1.15-3.26)$ & 0.013 & & 0.841 \\
\hline Tumour stroma percentage $(\leqslant 50 \% />50 \%)$ & $2.04(1.29-3.22)$ & 0.002 & $1.86(1.10-3.15)$ & 0.021 \\
\hline
\end{tabular}




\begin{tabular}{|c|c|c|c|c|}
\hline & \multicolumn{2}{|c|}{ Univariate analysis } & \multicolumn{2}{|c|}{ Multivariate analysis } \\
\hline & $\begin{array}{l}\text { Hazard ratio ( } 95 \% \\
\text { confidence interval) }\end{array}$ & $P$-value & $\begin{array}{l}\text { Hazard ratio ( } 95 \% \\
\text { confidence interval) }\end{array}$ & $P$-value \\
\hline Age $(\leqslant 50 />50$ years $)$ & $0.70(0.36-1.36)$ & 0.290 & & \\
\hline Size $(\leqslant 20 / 21-50 />50 \mathrm{~mm})$ & $2.32(1.25-4.31)$ & 0.007 & $2.47(1.23-4.95)$ & 0.011 \\
\hline Grade (I/IIIIIII) & $1.64(0.97-2.73)$ & 0.062 & & 0.217 \\
\hline Oestrogen receptor status (no/yes) & $0.71(0.36-1.40)$ & 0.325 & & \\
\hline Progesterone (no/yes) & $0.75(0.35-1.56)$ & 0.437 & & \\
\hline Her2 status (no/yes) & $2.11(1.03-4.31)$ & 0.040 & & 0.306 \\
\hline Lymphovascular invasion (no/yes) & $1.62(0.78-3.38)$ & 0.198 & & \\
\hline Tumour necrosis (no/yes) & $1.97(1.48-8.59)$ & 0.005 & $2.51(1.03-6.13)$ & 0.043 \\
\hline General inflammatory infiltrate (low/high) & $1.47(0.76-2.86)$ & 0.255 & & \\
\hline CD68 + macrophage infiltrate (tertiles) & $0.68(0.43-1.07)$ & 0.096 & & 0.313 \\
\hline CD4 + T-lymphocyte infiltrate (tertiles) & $0.88(0.59-1.32)$ & 0.520 & & \\
\hline CD8 + T-lymphocyte infiltrate (tertiles) & $0.89(0.59-1.33)$ & 0.558 & & \\
\hline $\begin{array}{l}\text { Loco-regional therapy (Lumpectomy + radiotherapy/ } \\
\text { mastectomy + radiotherapy) }\end{array}$ & $2.11(1.01-4.39)$ & 0.047 & & 0.188 \\
\hline Systemic adjuvant therapy (no/yes) & $1.18(0.57-2.42)$ & 0.657 & & \\
\hline Tumour stroma percentage ( $\leqslant 50 \% />50 \%)$ & $2.24(1.29-4.97)$ & 0.005 & $3.11(1.53-6.33)$ & 0.002 \\
\hline
\end{tabular}

Table 8. The relationship between clinicopathological characteristics and cancer-specific survival in patients with triple negative breast cancer $(n=151)$

\begin{tabular}{|c|c|c|c|c|}
\hline & \multicolumn{2}{|c|}{ Univariate analysis } & \multicolumn{2}{|c|}{ Multivariate analysis } \\
\hline & $\begin{array}{l}\text { Hazard ratio ( } 95 \% \\
\text { confidence interval) }\end{array}$ & $P$-value & $\begin{array}{l}\text { Hazard ratio( } 95 \% \\
\text { confidence interval) }\end{array}$ & $P$-value \\
\hline Age $(\leqslant 50 />50$ years $)$ & $1.23(0.69-2.18)$ & 0.475 & & \\
\hline Size $(\leqslant 20 / 21-50 />50 \mathrm{~mm})$ & $3.10(1.91-5.04)$ & $<0.001$ & $2.53(1.52-4.21)$ & $<0.001$ \\
\hline Involved lymph node $(0 / 1-3 />3)$ & $1.91(1.34-2.71)$ & $<0.001$ & $1.64(1.15-2.34)$ & 0.007 \\
\hline Grade (I/II/III) & $0.97(0.54-1.74)$ & 0.916 & & \\
\hline Lymphovascular invasion (no/yes) & $2.11(1.20-3.70)$ & 0.009 & & 0.337 \\
\hline Tumour necrosis (no/yes) & $4.63(1.66-12.88)$ & $<0.001$ & $3.99(1.43-11.13)$ & 0.008 \\
\hline General inflammatory infiltrate (low/high) & $0.84(0.47-1.48)$ & 0.534 & & \\
\hline CD68 + macrophage infiltrate (tertiles) & $0.79(0.54-1.16)$ & 0.227 & & \\
\hline CD4 + T-lymphocyte infiltrate (tertiles) & $0.85(0.60-1.19)$ & 0.342 & & \\
\hline CD8 + T-lymphocyte infiltrate (tertiles) & $0.76(0.55-1.05)$ & 0.098 & & 0.253 \\
\hline $\begin{array}{l}\text { Loco-regional therapy (Lumpectomy + radiotherapy/ } \\
\text { mastectomy + radiotherapy) }\end{array}$ & $2.38(1.24-4.58)$ & 0.009 & & 0.176 \\
\hline Adjuvant therapy (no/yes) & $1.00(0.48-2.02)$ & 0.997 & & \\
\hline Tumour stroma percentage $(\leqslant 50 \% />50 \%)$ & $1.06(1.03-1.12)$ & 0.035 & & 0.151 \\
\hline
\end{tabular}

due to treatment regimen undertaken; though, in both studies patients did not receive neoadjuvant treatment. Irrespective of this, previous work has not determined whether the effect of an expanded tumour stroma on survival was independent of host inflammatory responses and other components of the tumour microenvironment.

Although the inter-relationships between the tumour stroma, tumour microenvironment and gross pathological characteristics are likely complex, the TSP remained independently and strongly associated with reduced cancer-specific survival. These results confirm the importance of tumour-host factors, such as the tumour microenvironment, in determining oncological outcome.
In particular, node-negative patients with high TSP had a more than two-fold higher risk of breast cancer death compared with those with low TSP, independent and comparable with that of tumour size, lymph node status, grade and necrosis. Furthermore, survival was also significantly shorter in patients who received adjuvant therapy for high-TSP tumours. Thus, in addition to identifying high-risk patients, TSP may also select patients less likely to benefit from standard therapy and who should be considered for additional adjunctive treatment, potentially targeted at the stroma itself (Engels et al, 2012). These results confirm the importance of tumour-host factors, such as the tumour microenvironment in determining oncological outcome. 
Despite recognition of the importance of the tumour stroma in cancer progression, its relationship with other components of the tumour microenvironment has yet to be fully characterised. In the present study, increased amount of stroma was associated with a weaker peritumoural inflammatory infiltrate, as measured by the Klintrup-Mäkinen score and by macrophages and T-cell subtypes. This is consistent with the recent observation that a high TSP trended toward a low peritumoural inflammatory infiltrate in patients with colorectal cancer (Park et al, 2014); however, the underlying mechanism is still unclear. In the present study, amount of tumour stroma was not associated with hormone receptors or proliferative marker such as Ki67, although it is of interest that Ki67 was recently shown to be significantly associated with tumour inflammatory infiltrates (Mohammed et al, 2012a,b,c,d).

It has previously been proposed that the tumour stroma may prevent effective tumour infiltration by immune cells (Ueno et al, 2004). The results from cell line experiments would also support these findings, namely that fibroblasts and myofibroblasts can modulate the ability of lymphocytes and macrophages to invade a tumour and may prevent penetration of immune cells within tumours, creating a physical barrier against an immune reaction while promoting tumour growth and progression, due to their contractile properties and their associated extracellular matrix (Lieubeau et al, 1999).

In the present study, although the cell markers of both innate and adaptive immune cells were examined in the present study, the effect of TSP on survival remained independent of local inflammatory responses, suggesting the presence of other mechanisms rather than a direct effect on immune cells. Indeed, tumour stroma may promote the development of a pro-tumour rather than anti-tumour immune infiltrate (Fridman et al, 2011). Stromal fibroblasts may also induce suppression of the immune response and produce immunosuppressive molecules such as TGF- $\beta$ and VEGF, suggesting that CAFs may promote cancer immunoescape (Yaguchi et al, 2011; Engels et al, 2012). This may also implicate certain cell signalling pathways such as the common cell signalling pathway associated with inflammation; the JAK-State pathway (Yu et al, 2007, 2009). Therefore, further characterisation of the tumour inflammatory cell infiltrate and their association with tumour stroma and JAK-State signalling is warranted.

In the present study, amount of tumour stroma was not associated with hormone receptors or the proliferative marker Ki67. However, it is of interest that the hormone receptors were recently shown to be significantly associated with tumour inflammatory infiltrates. Patients with high-grade general inflammatory infiltrate were more likely to have ER-negative and PRnegative tumours. The expression of ER/PR was directly associated with the percentage of tumour lymphocyte infiltrate and inversely associated with $\mathrm{CD} 68+, \mathrm{CD} 8+$ and $\mathrm{CD} 138+$ infiltrates. Similarly, the expression of HER-2 was directly associated with $\mathrm{CD} 8+$ and inversely associated with $\mathrm{CD} 138+$ infiltrates (Baker et al, 2011; Mohammed et al, 2012c, 2013).

A potential limitation of the present study was that direct investigation of the effect of tumour stroma on the infiltration of inflammatory cells was not carried. This would require either cell line or animal models. Although cell line or animal models do have the advantage of allowing direct investigation of the effect of tumour stroma on inflammatory cell infiltration, they often lack clinical relevance to the patient with breast cancer with the consequent slow progress on immunotherapy for breast cancer. The present study highlights the importance of the amount of tumour stroma on immunological response in patients with primary operable ductal breast cancer.

In conclusion, the results of the present study show that a high TSP in primary operable invasive ductal breast cancer was associated with recurrence and shorter long-term survival.
Implementing this simple and reproducible parameter in routine pathological examination may help optimise risk stratification in patients with invasive ductal breast cancer. The present study findings suggest that high TSP enables tumour cells to evade the immune response and promote tumour progression.

\section{ACKNOWLEDGEMENTS}

We gratefully acknowledge the Ministry of Higher Education-Libya for funding this work. We also thank Clare Orange, who provided technical assistance.

\section{REFERENCES}

Baker K, Lachapelle J, Zlobec I, Bismar TA, Terracciano L, Foulkes WD (2011) Prognostic significance of CD8 + T lymphocytes in breast cancer depends upon both oestrogen receptor status and histological grade. Histopathology 58(7): 1107-1116.

Cirri P, Chiarugi P (2012) Cancer-associated-fibroblasts and tumour cells: a diabolic liaison driving cancer progression. Cancer Metastasis Rev 31(1-2): 195-208.

Colotta F, Allavena P, Sica A, Garlanda C, Mantovani A (2009) Cancer-related inflammation, the seventh hallmark of cancer: links to genetic instability. Carcinogenesis 30(7): 1073-1081.

de Kruijf EM, van Nes JG, Van de Velde CJ, Putter H, Smit VT, Liefers GJ, Kuppen PJ, Tollenaar RA, Mesker WE (2011) Tumor-stroma ratio in the primary tumor is a prognostic factor in early breast cancer patients, especially in triple-negative carcinoma patients. Breast Cancer Res Treat 125(3): 687-696.

Dekker TJ, van de Velde CJ, van Pelt GW, Kroep JR, Julien JP, Smit VT, Tollenaar RA, Mesker WE (2013) Prognostic significance of the tumorstroma ratio: validation study in node-negative premenopausal breast cancer patients from the EORTC perioperative chemotherapy (POP) trial (10854). Breast Cancer Res Treat 139(2): 371-379.

Engels B, Rowley DA, Schreiber H (2012) Targeting stroma to treat cancers. Semin Cancer Biol 22(1): 41-49.

Ferlay J, Parkin D, Steliarova-Foucher E (2010) Estimates of cancer incidence and mortality in Europe in 2008. Eur J Cancer 46: 765-781.

Fridman WH, Galon J, Pages F, Tartour E, Sautès-Fridman C, Kroemer G (2011) Prognostic and predictive impact of intra- and peritumoral immune infiltrates. Cancer Res 71(17): 5601-5605.

Hanahan D, Weinberg RA (2011) Hallmarks of cancer: the next generation. Cell 144(5): 646-674.

Health and Consumer Protection Directorate-General (2006) European Guidelines for Quality Assurance in Breast Cancer Screening and Diagnosis. European Communities: Luxembourg.

$\mathrm{Hu}$ M, Polyak K (2008) Microenvironmental regulation of cancer development. Curr Opin Genet Dev 18(1): 27-34.

Huijbers A, RA Tollenaar, v Pelt GW, Zeestraten EC, Dutton S, McConkey CC, Domingo E, VT Smit, Midgley R, Warren BF, Johnstone EC, Kerr DJ, Mesker WE (2013) The proportion of tumor-stroma as a strong prognosticator for stage II and III colon cancer patients: validation in the VICTOR trial. Ann Oncol 24(1): 179-185.

Ikpatt O, Ndoma-Egba R, Collan Y (2002) Prognostic value of necrosis in Nigerian breast cancer. Adv Clin Pathol 6(1): 31-37.

Kim JB, Stein R, O'Hare MJ (2005) Tumour-stromal interactions in breast cancer: the role of stroma in tumourigenesis. Tumor Biol 26(4): 173-185.

Klintrup K, Makinen JM, Kauppila S, Vare PO, Melkko J, Tuominen H, Tuppurainen K, Makela J, Karttunen TJ, Makinen MJ (2005) Inflammation and prognosis in colorectal cancer. Eur JCancer 41(17): 2645-2654.

Lieubeau B, Heymann MF, Henry F, Barbieux I, Meflah K, Grégoire M (1999) Immunomodulatory effects of tumorassociated fibroblasts in colorectaltumor development. Int J Cancer 81(4): 629-636.

Mesker WE, Junggeburt JM, Szuhai K, de Heer P, Morreau H, Tanke HJ, Tollenaar RA (2007) The carcinoma-stromal ratio of colon carcinoma is an independent factor for survival compared to lymph node status and tumor stage. Cell Oncol 29(5): 387-398.

Mirza AN, Mirza NQ, Vlastos G, Singletary ES (2002) Prognostic factors in node-negative breast cancer: a review of studies with sample size more than 200 and follow-up more than 5 years. Ann Surg 235(1): 10-26. 
Mohammed ZM, Edwards J, Orange C, Mallon E, Doughty JC, McMillan DC, Going JJ (2012a) Breast cancer outcomes by steroid hormone receptor status assessed visually and by computer image analysis. Histopathology 61(2): 283-292.

Mohammed ZM, Going JJ, McMillan DC, Orange C, Mallon E, Doughty JC, Edwards J (2012b) Comparison of visual and automated assessment of HER2 status and their impact on outcome in primary operable invasive ductal breast cancer. Histopathology 61(4): 675-684.

Mohammed ZM, Going J, Edwards J, Elsberger B, Doughty J, McMillan D (2012c) The relationship between components of tumour inflammatory cell infiltrate and clinicopathological factors and survival in patients with primary operable invasive ductal breast cancer. Br J Cancer 107(5): 864-873.

Mohammed ZM, Going JJ, Edwards J, Elsberger B, McMillan DC (2013) The relationship between lymphocyte subsets, hormone status and clinicopathological determinants of survival in patients with primary operable invasive ductal breast cancer. Br J Cancer 109(6): $1676-1684$.

Mohammed ZM, McMillan DC, Elsberger B, Going JJ, Orange C, Mallon E, Doughty JC, Edwards J (2012d) Comparison of visual and automated assessment of Ki-67 proliferative activity and their impact on outcome in primary operable invasive ductal breast cancer. $\mathrm{Br} J$ Cancer 106(2): 383-388.

Moorman AM, Vink R, Heijmans HJ, van der Palen J, Kouwenhoven EA (2012) The prognostic value of tumour-stroma ratio in triple-negative breast cancer. Eur J Surg Oncol 38(4): 307-313.

Park J, Richards C, McMillan D, Horgan P, Roxburgh C (2014) The relationship between tumour stroma percentage, the tumour microenvironment and survival in patients with primary operable colorectal cancer. Ann Oncoly 25(3): 644-651.
Courrech Staal EF, Wouters MW, van Sandick JW, Takkenberg MM, Smit VT, Junggeburt JM, Spitzer-Naaykens JM, Karsten T, Hartgrink HH, Mesker WE, Tollenaar RA (2010) The stromal part of adenocarcinomas of the oesophagus: Does it conceal targets for therapy? Eur J Cancer 46(4): 720-728.

Ueno H, Jones AM, Wilkinson KH, Jass JR, Talbot IC (2004) Histological categorisation of fibrotic cancer stroma in advanced rectal cancer. Gut 53: 581-586.

Wang K, Ma W, Wang J, Yu L, Zhang X, Wang Z, Tan B, Wang N, Bai B, Yang S, Liu H, Zhu S, Cheng Y (2012) Tumor-stroma ratio is an independent predictor for survival in esophageal squamous cell carcinoma. J Thorac Oncol 7(9): 1457-1461.

West NP, Dattani M, McShane P, Hutchins G, Grabsch J, Mueller W, Treanor D, Quirke P, Grabsch H (2010) The proportion of tumour cells is an independent predictor for survival in colorectal cancer patients. Br J Cancer 102(10): 1519-1523.

Yaguchi T, Sumimoto H, Kudo-Saito C, Tsukamoto N, Ueda R, Iwata-Kajihara T, Nishio H, Kawamura N, Kawakami Y (2011) The mechanisms of cancer immunoescape and development of overcoming strategies. Int J Hematol 93(3): 294-300.

Yu H, Kortylewski M, Pardoll D (2007) Crosstalk between cancer and immune cells: role of STAT3 in the tumour microenvironment. Nat Rev Immunol 7(1): 41-51.

Yu H, Pardoll D, Richard J (2009) STATs in cancer inflammation and immunity: a leading role for STAT3. Nat Rev Cancer 9(11): 798-809.

This work is published under the standard license to publish agreement. After 12 months the work will become freely available and the license terms will switch to a Creative Commons AttributionNonCommercial-Share Alike 3.0 Unported License. 